MATHEMATICS OF COMPUTATION

Volume 75, Number 255, July 2006, Pages 1367-1382

S 0025-5718(06)01830-8

Article electronically published on February 22, 2006

\title{
LIMITING SET OF SECOND ORDER SPECTRA
}

\author{
LYONELL BOULTON
}

\begin{abstract}
Let $M$ be a self-adjoint operator acting on a Hilbert space $\mathcal{H}$. A complex number $z$ is in the second order spectrum of $M$ relative to a finitedimensional subspace $\mathcal{L} \subset \operatorname{Dom} M^{2}$ iff the truncation to $\mathcal{L}$ of $(M-z)^{2}$ is not invertible. This definition was first introduced in Davies, 1998, and according to the results of Levin and Shargorodsky in 2004, these sets provide a method for estimating eigenvalues free from the problems of spectral pollution. In this paper we investigate various aspects related to the issue of approximation using second order spectra. Our main result shows that under fairly mild hypothesis on $M$, the uniform limit of these sets, as $\mathcal{L}$ increases towards $\mathcal{H}$, contain the isolated eigenvalues of $M$ of finite multiplicity. Therefore, unlike the majority of the standard methods, second order spectra combine nonpollution and approximation at a very high level of generality.
\end{abstract}

\section{INTRODUCTION}

Let $M$ be a self-adjoint operator acting on an infinite-dimensional Hilbert space $\mathcal{H}$ and let $\lambda$ be an isolated eigenvalue of $M$ of finite multiplicity. Suppose we intend to estimate $\lambda$ numerically. A natural method, which is already over one hundred years old, is to truncate $M$ by taking an orthonormal basis $\left\{\phi_{j}\right\}_{j=1}^{\infty}$ of $\mathcal{H}$ contained in the (operator or form) domain of $M$ and compute the eigenvalues of the $n \times n$ matrix $M_{n}=\left(\left\langle M \phi_{j}, \phi_{k}\right\rangle\right)_{j, k=1}^{n}$ for large $n$, expecting that some of these eigenvalues would be close to $\lambda$. Unfortunately, an ill chosen $\left\{\phi_{j}\right\}_{j=1}^{\infty}$ will result in either of the following difficulties:

- Lack of approximation: no eigenvalue of $M_{n}$ is close to $\lambda$. This happens when $M$ is unbounded.

- Spurious eigenvalues: or spectral pollution, $M_{n}$ has many eigenvalues unrelated to the spectrum of $M$ in a large neighbourhood of $\lambda$. This occurs when $\lambda$ is in a gap, i.e., in between two points of the essential spectrum of $M$.

It is well known that if $M$ is an operator bounded below and $\lambda<\min \left[\operatorname{Spec}_{\text {ess }} M\right]$, then the Rayleigh-Ritz theorem provides satisfactory procedures to deal with these two issues. For instance, suppose that $\lambda$ is the first eigenvalue of $M$ and it is

Received by the editor July 16, 2003 and, in revised form, April 18, 2005.

2000 Mathematics Subject Classification. Primary 47B36; Secondary 47B39, 81-08.

Key words and phrases. Second order spectrum, projection methods, spectral pollution, numerical approximation of the spectrum.

(C)2006 American Mathematical Society Reverts to public domain 28 years from publication 
nondegenerate. If $\left\{\phi_{j}\right\}_{j=1}^{\infty}$ is such that

$$
M_{n}\left(\sum_{j=1}^{n}\left\langle\psi, \phi_{j}\right\rangle \phi_{j}\right) \rightarrow \lambda \psi \text { as } n \rightarrow \infty \text { whenever } M \psi=\lambda \psi,
$$

then the first eigenvalue of $M_{n}$ converges to $\lambda$ (i.e., we achieve approximation) and the second eigenvalue of $M_{n}$, counting multiplicity, cannot be smaller than the second eigenvalue of $M$ (i.e., no chance of spurious eigenvalues). Condition (11) is useful in applications, because it can sometimes be verified on abstract grounds without having much information about the eigenfunctions $\psi$; cf. [13, Theorem XIII.4].

In contrast, when $\lambda$ is in between two points of the essential spectrum, it is well known that pollution can arise. In fact, for each $\mu \in \mathbb{R} \backslash$ Spec $M$ lying between two points of the essential spectrum, there exists an orthonormal basis $\left\{\phi_{j}\right\}_{j=1}^{\infty}$ and a subsequence $n(1)<n(2)<\ldots$, such that $\mu \in \operatorname{Spec} M_{n(k)}$ for all $k \in \mathbb{N}$ (see [7). There is a large amount of literature devoted to the study of this problem in application; we refer to 5 and 7 , and the references therein, for a more complete account of the matter.

In order to find eigenvalues in gaps of the essential spectrum, one can appeal to the following geometrically motivated idea (see [5] and 9]). Suppose $a<\zeta<b$, such that $a, b \in \operatorname{Spec}_{\text {ess }} M$ but $(a, b) \cap \operatorname{Spec}_{\text {ess }} M=\varnothing$. Then $(M-\zeta)^{2}$ is bounded below by zero and the eigenvalues below $\operatorname{Spec}_{\text {ess }}(M-\zeta)^{2}$ are the $(\lambda-\zeta)^{2} \geq 0$, where $\lambda$ is an eigenvalue of $M$ inside $(a, b)$. Hence by applying the Rayleigh-Ritz principle to $(M-\zeta)^{2}$, we might be able to estimate (up to a square root ambiguity) the eigenvalue $\lambda$. If, for instance, $(M-\zeta)^{2}$ satisfies (1), from the first eigenvalue of its matrix truncations we may estimate the closest eigenvalue of $M$ to $\zeta$ in $(a, b)$.

Instead of looking for the eigenvalues close to zero for a truncation of $(M-\zeta)^{2}$, we can also consider finding $z$ such that the truncation of $(M-z)^{2}$ is singular. Denote by $\mathcal{L}_{n}$ the $\operatorname{Span}\left\{\phi_{j}\right\}_{j=1}^{n}$ and by $P_{n}$ the orthogonal projection onto $\mathcal{L}_{n}$. Assume that $\mathcal{L}_{n} \subset$ Dom $M^{2}$. We say that $z \in \mathbb{C}$ is an element of the second order spectrum of $M$ relative to $\mathcal{L}_{n}$, denoted below by $\operatorname{Spec}_{2}\left(M \mid \mathcal{L}_{n}\right)$, if and only if $\operatorname{det}\left[P_{n}(M-z)^{2} \mid \mathcal{L}_{n}\right]=$ 0 . This definition was first introduced in [4 by Davies. Levitin and Shargorodsky have recently proposed (see [7) using second order spectra to approximate isolated eigenvalues inside spectral gaps, on the grounds that they are free from pollution. To be precise, if $z \in \operatorname{Spec}_{2}\left(M \mid \mathcal{L}_{n}\right)$, then

$$
[\operatorname{Re} z-|\operatorname{Im} z|, \operatorname{Re} z+|\operatorname{Im} z|] \cap \operatorname{Spec} M \neq \varnothing
$$

(cf. [7] and [14]). In other words, a point in $\operatorname{Spec}_{2}\left(M \mid \mathcal{L}_{n}\right)$ is close to the real axis only when it is also close to the spectrum. Therefore numerical procedures based on second order spectrum never lead to spurious spectrum.

Although the bound (2) does not guarantee that any point at all in the spectrum is estimated for large $n$, some numerical experiments performed in 77 and the results of 2 indicate that approximation occurs in test models.

In this paper we discuss rigorously various aspects of the question of approximation in the method considered by Levitin and Shargorodsky. For this, we study the uniform limiting set

$$
\Lambda:=\mathrm{u}-\lim _{n \rightarrow \infty} \operatorname{Spec}_{2}\left(M \mid \mathcal{L}_{n}\right)=\left\{z \in \mathbb{C}: z_{n} \rightarrow z, z_{n} \in \operatorname{Spec}_{2}\left(M \mid \mathcal{L}_{n}\right)\right\} .
$$


The interest in $\Lambda$ is clear; the points in $\operatorname{Spec} M$ which are estimated by $\operatorname{Spec}_{2}\left(M \mid \mathcal{L}_{n}\right)$ for $n$ large are those in $\Lambda \cap \mathbb{R}$. The following assumption on $\lambda$ and $P_{n}$ will be crucial in our subsequent analysis:

$$
\begin{aligned}
& \text { if } M \psi=\lambda \psi \text {, then }\left\|P_{n} M P_{n} \psi-\lambda \psi\right\| \rightarrow 0 \\
& \text { and }\left\|P_{n} M^{2} P_{n} \psi-\lambda^{2} \psi\right\| \rightarrow 0, \text { as } n \rightarrow \infty .
\end{aligned}
$$

Our principal task will be to show the following.

Theorem 1. If $\lambda$ is an isolated eigenvalue of finite multiplicity of $M$ and $(\mathrm{H})$ holds, then $\lambda \in \Lambda$.

Obviously any bounded operator satisfies $(\mathrm{H})$, and note the analogy between $(\mathrm{H})$ and (1) in the general case. We will discuss in Section 5 how to verify this hypothesis for unbounded operators without having much information about $\psi$. We remark that $(\mathrm{H})$ is similar to a known condition for spectral approximation in the self-adjoint case given in [11, Theorems VIII.24-25].

We should emphasize the relevance of this result. In many applications, the essential spectrum can be found analytically, whereas the isolated eigenvalues of finite multiplicity are the ones that should be estimated numerically. Theorem 1 shows that second order relative spectra provide a method which will give convergence to these eigenvalues. Furthermore, in conjunction with (2), it is ensured that the method will not pollute, no matter the location of these eigenvalues. We are not aware of any other technique that combines approximation and nonpollution at such level of generality.

In Section 2 we keep close to the ideas of [4]. We characterize $\operatorname{Spec}_{2}\left(M \mid \mathcal{L}_{n}\right)$ and the set $\Lambda$ in terms of resolvent norms. This characterization will be important in Section 3, where we prove Theorem 1 In Section 4 we discuss in detail a simple model bounded operator, for which $\Lambda$ can be found explicitly. In order to test Theorem 1 in concrete situations, we also include some experimental numerical outputs for rank one perturbations of this model. Finally Section 5 is devoted to discussing the verification of condition $(\mathrm{H})$ for concrete unbounded situations.

\section{SECOND ORDER SPECTRUM AND NORM OF INVERSES}

The sequence of subspaces $\mathcal{L}_{n}$ does not necessarily need to be nested in order to accomplish approximation. Below $P_{n}$ denotes a family of orthogonal projections and $\mathcal{L}_{n}:=\operatorname{Ran} P_{n} \subset \operatorname{Dom} M^{2}$, such that $\operatorname{dim} \mathcal{L}_{n}=n$ and $P_{n} x \rightarrow x$ for all $x \in \mathcal{H}$. For the linear operator $H$, we shall often denote by $H_{n}$ the truncation $P_{n} H \mid \mathcal{L}_{n}$. By $H_{n}^{-1}$ we shall always mean the inverse of $H_{n}$ within $\mathcal{L}_{n}$. If $H_{n}$ is not invertible we allow the abuse of notation $\left\|H_{n}^{-1}\right\|=\infty$.

From the definition it is easy to deduce that $\operatorname{Spec}_{2}\left(M \mid \mathcal{L}_{n}\right)$ is a set of at most $2 n$ different complex numbers. In general these points do not intersect the real line, unless $\mathcal{L}_{n}$ contains an eigenfunction of $M$. Since

$$
\overline{\operatorname{det} P_{n}(M-z)^{2} \mid \mathcal{L}_{n}}=\operatorname{det} P_{n}(M-\bar{z})^{2} \mid \mathcal{L}_{n},
$$

$\operatorname{Spec}_{2}\left(M \mid \mathcal{L}_{n}\right)$ is symmetric with respect to $\mathbb{R}$. The limiting set $\Lambda$ might or might not contain nonreal points. Reference [2] is largely devoted to showing that $\Lambda \subseteq$ Spec $M$, when $M$ is a discrete Schrödinger operator whose potential decays fast at infinity. On the other hand, in the example we discuss in Section $4, \Lambda$ is equal to the unit circle. 
In order to describe properties of $\Lambda$, it is useful to characterize the points in $\operatorname{Spec}_{2}\left(M \mid \mathcal{L}_{n}\right)$ as the zeros of a certain Lipschitz continuous function $\sigma_{n}(z)$. Davies in 4, and Davies and Plum in [5] study a procedure for computing eigenvalues of bounded self-adjoint operators, based on evaluating $\sigma_{n}(z)^{\frac{1}{2}}$ at $z \in \mathbb{R}$. Let

$$
\sigma_{n}(z):=\inf \left\{\frac{\left\|P_{n}(M-z)^{2} \mid \mathcal{L}_{n} v\right\|}{\|v\|}: 0 \neq v \in \mathcal{L}_{n}\right\}
$$

If $z \notin \operatorname{Spec}_{2}\left(M \mid \mathcal{L}_{n}\right)$, then $\sigma_{n}(z)^{-1}=\left\|\left[P_{n}(M-z)^{2} \mid \mathcal{L}_{n}\right]^{-1}\right\|$. Moreover, $\sigma_{n}(z)=0$ if and only if $z \in \operatorname{Spec}_{2}\left(M \mid \mathcal{L}_{n}\right)$. Both assertions are a direct consequence of the definition.

The following result shows that, in order to find the zeros of $\sigma_{n}$, we can start from an initial guess and move in steps towards a local minimum.

Lemma 2. The function $\sigma_{n}(z)$ is a nonnegative Lipschitz continuous function of the variable $z$. Its only local minima are the points where it vanishes.

Proof. We follow closely [4, Theorem 21]. If $|w-z|<\varepsilon$, then

$$
\begin{aligned}
& \sigma_{n}(z)=\inf \frac{\left\|P_{n}(M-z)^{2} \mid \mathcal{L}_{n} v\right\|}{\|v\|} \\
& \quad \leq \inf \frac{\left\|P_{n}(M-w)^{2}\left|\mathcal{L}_{n} v\|+|w-z|\| P_{n}(2 M-z-w)\right| \mathcal{L}_{n} v\right\|}{\|v\|} \\
& \quad \leq \sigma_{n}(w)+|w-z| \sup \frac{\left\|\left(2 M_{n}-z-w\right) v\right\|}{\|v\|} \\
& \quad \leq \sigma_{n}(w)+|w-z| c,
\end{aligned}
$$

where $c>0$ can be chosen independent of $\varepsilon$. This ensures the Lipschitz continuity of $\sigma_{n}$.

On the other hand, given $z_{0} \in \mathbb{C} \backslash \operatorname{Spec}_{2}\left(M \mid \mathcal{L}_{n}\right)$, choose $u, v \in \mathcal{L}_{n}$ vectors of norm 1 such that

$$
\sigma_{n}\left(z_{0}\right)^{-1}=\left|\left\langle\left[P_{n}\left(M-z_{0}\right)^{2} \mid \mathcal{L}_{n}\right]^{-1} u, v\right\rangle\right| .
$$

The function $\left\langle\left[P_{n}(M-z)^{2} \mid \mathcal{L}_{n}\right]^{-1} u, v\right\rangle$ is analytic in $z \in \mathbb{C} \backslash \operatorname{Spec}_{2}\left(M \mid \mathcal{L}_{n}\right)$. We claim that it is not constant. If such a claim is true, then there always exists $z$ in any neighbourhood of $z_{0}$ such that

$$
\begin{aligned}
\sigma_{n}(z)^{-1} & \geq\left|\left\langle\left[P_{n}(M-z)^{2} \mid \mathcal{L}_{n}\right]^{-1} u, v\right\rangle\right| \\
& >\left|\left\langle\left[P_{n}\left(M-z_{0}\right)^{2} \mid \mathcal{L}_{n}\right]^{-1} u, v\right\rangle\right| \\
& =\sigma_{n}\left(z_{0}\right)^{-1},
\end{aligned}
$$

ensuring the second assertion of the lemma. In order to prove our claim, first note that

$$
\begin{aligned}
& \left|\left\langle\left[P_{n}(M-z)^{2} \mid \mathcal{L}_{n}\right]^{-1} u, v\right\rangle\right| \leq\left\|\left[P_{n}(M-z)^{2} \mid \mathcal{L}_{n}\right]^{-1}\right\| \\
& \quad=|z|^{-2}\left\|\left[z^{-2} P_{n} M^{2}\left|\mathcal{L}_{n}-2 z^{-1} P_{n} M\right| \mathcal{L}_{n}+1\right]^{-1}\right\| .
\end{aligned}
$$

Then, if $|z|>3 \max \left\{\left\|P_{n} M^{2}\left|\mathcal{L}_{n}\|,\| P_{n} M\right| \mathcal{L}_{n}\right\|, 1\right\}$,

$$
\left\|z^{-2} P_{n} M^{2}\left|\mathcal{L}_{n}-2 z^{-1} P_{n} M\right| \mathcal{L}_{n}\right\|<7 / 9<1,
$$

and hence,

$$
\left|\left\langle\left[P_{n}(M-z)^{2} \mid \mathcal{L}_{n}\right]^{-1} u, v\right\rangle\right| \leq(9 / 2)|z|^{-2} \rightarrow 0
$$

as $|z| \rightarrow \infty$. Thus necessarily the above-mentioned function is not constant. 
In conjunction with $\Lambda$, it is natural to consider the set of asymptotic zeros of the family $\left\{\sigma_{n}\right\}$,

$$
\Sigma \equiv \Sigma(M):=\left\{z \in \mathbb{C}: \sigma_{n}(z) \rightarrow 0, n \rightarrow \infty\right\} .
$$

If $M$ is bounded, this set contains the limiting set $\Lambda$. Indeed, suppose that $z \notin \Sigma$. Then there is a subsequence $n(j)$ and $c>0$, such that $P_{n(j)}(M-z)^{2} \mid \mathcal{L}_{n(j)}$ are invertible for all $j \in \mathbb{N}$ and

$$
c \geq \sigma_{n(j)}^{-1}(z)=\left\|\left[P_{n(j)}(M-z)^{2} \mid \mathcal{L}_{n(j)}\right]^{-1}\right\| .
$$

By virtue of (3), for $|z-w|<\varepsilon$,

$$
\sigma_{n(j)}(z) \leq \sigma_{n(j)}(w)+|w-z| c_{1},
$$

where $c_{1}>0$ can be chosen independent of $\varepsilon$ and $j$ (as $M$ is bounded, $c_{1}$ does not depend on $j$ ). By letting $\varepsilon$ be small enough, we can find $c_{2}>0$ such that $\sigma_{n(j)}(w) \geq c_{2}$ whenever $|w-z|<\varepsilon$. Thus

$$
\bigcup_{j=1}^{\infty} \operatorname{Spec}_{2}\left(M \mid \mathcal{L}_{n(j)}\right) \cap\{|z-w|<\varepsilon\}=\varnothing,
$$

so therefore $z \notin \Lambda$.

Furthermore, whenever $M$ is bounded, $\Sigma \cap \mathbb{R}=\operatorname{Spec} M$. Indeed, if $\lambda \in \operatorname{Spec} M$, for each $k>0$ there is $\psi_{k} \in \mathcal{H},\left\|\psi_{k}\right\|=1$, such that $\left\|(M-\lambda)^{2} \psi_{k}\right\|<1 / k$. Then

$$
\lim _{n \rightarrow \infty} \frac{\left\|P_{n}(M-\lambda)^{2} P_{n} \psi_{k}\right\|}{\left\|P_{n} \psi_{k}\right\|}<1 / k
$$

and so $\sigma_{n}(\lambda) \rightarrow 0$ as $n \rightarrow \infty$. Conversely, note that

$$
\left\langle P_{n}(M-z)^{2} \mid \mathcal{L}_{n} v, v\right\rangle=\left\langle(M-z)^{2} v, v\right\rangle
$$

for all $v \in \mathcal{L}_{n},\|v\|=1$. Then, if $\lambda \in \mathbb{R}$ but $\lambda \notin \operatorname{Spec} M$,

$$
\operatorname{Num} P_{n}(M-\lambda)^{2} \mid \mathcal{L}_{n} \subset \operatorname{Num}(M-\lambda)^{2} \subset[b, \infty)
$$

for some constant $b>0$ independent of $n$. Hence

$$
\sigma_{n}(\lambda) \geq \operatorname{dist}\left[0, \operatorname{Num} P_{n}(M-\lambda)^{2} \mid \mathcal{L}_{n}\right] \geq b,
$$

so that $\lambda \notin \Sigma \cap \mathbb{R}$. This observation is crucial in the method of Davies and Plum mentioned earlier. Note that the direction $\Sigma \cap \mathbb{R} \subseteq$ Spec $M$ does not require $M$ to be bounded, whereas the other inclusion becomes a slightly more involved problem in general. Here and below "Num" denotes numerical range.

It would be of obvious interest to find hypotheses which guarantee $\Lambda=\Sigma$. We show that the equality holds at least in particular cases. Below and elsewhere we denote by $\mathbb{T}$ the unit circle.

Proposition 3. If Spec $M$ consists of two points $a<b$, then

$$
\Sigma=\Lambda \subseteq\{z \in \mathbb{C}:|z-(a+b) / 2|=(b-a) / 2\} .
$$

Proof. Since

$$
\operatorname{Spec}_{2}\left(\alpha M+\beta \mid \mathcal{L}_{n}\right)=\alpha \operatorname{Spec}_{2}\left(M \mid \mathcal{L}_{n}\right)+\beta
$$

and $\Sigma(\alpha M+\beta)=\alpha \Sigma(M)+\beta$ for scalars $\alpha$ and $\beta$, it is enough to show the desired property for the case Spec $M=\{ \pm 1\}$. By virtue of the hypothesis, it is clear that $z=0$ can neither be in $\Sigma$ nor in any of the $\operatorname{Spec}_{2}\left(M \mid \mathcal{L}_{n}\right)$, so below we assume $z \neq 0$. 
The latter assumption on $M$ yields $M^{2}=\mathrm{Id}$. Then, since

$$
P_{n}(M-z)^{2} \mid \mathcal{L}_{n}=2 z\left[\left(z^{-1}+z\right) / 2-P_{n} M \mid \mathcal{L}_{n}\right],
$$

$z \in \operatorname{Spec}_{2}\left(M \mid \mathcal{L}_{n}\right)$ if and only if

$$
\left(z^{-1}+z\right) / 2 \in \operatorname{Spec} M_{n} \subset \operatorname{Num} M=[-1,1] .
$$

Hence all the second order relative spectra of $M$ are contained in $\mathbb{T}$. This shows the second relation.

We already saw that $\Lambda \subseteq \Sigma$ in general. In order to show the reverse inclusion, assume that $z \notin \Lambda$. Then there is a subsequence $n(j)$ and $\delta>0$, such that

$$
\bigcup_{j=1}^{\infty} \operatorname{Spec}_{2}\left(M \mid \mathcal{L}_{n(j)}\right) \cap\{|z-w|<\delta\}=\varnothing .
$$

By virtue of (5),

$$
\sigma_{n(j)}(z)=2|z| \operatorname{dist}\left[\left(z^{-1}+z\right) / 2, \operatorname{Spec} M_{n(j)}\right] .
$$

But if $\left(w^{-1}+w\right) / 2$ is in the spectrum of $M_{n(j)}$,

$$
\begin{aligned}
\left|\left(z^{-1}+z\right) / 2-\left(w^{-1}+w\right) / 2\right| & =|z|^{-1}|w-z|\left|w^{-1}-z\right| \\
& \geq|z|^{-1} \delta^{2} .
\end{aligned}
$$

Hence $\sigma_{n(j)}(z) \geq c>0$ for all $j \in \mathbb{N}$ and so $z \notin \Sigma$. This shows Proposition 3 ,

In application it is of interest to understand how the second order spectrum and its limiting set change under compact perturbations. Let $H$ be a bounded not necessarily self-adjoint operator and let $K$ be a compact operator. It is well known that $\left\|H_{n}^{-1}\right\|$ is uniformly bounded for all $n$ large and $H+K$ is invertible, if and only if $\left\|(H+K)_{n}^{-1}\right\|$ is uniformly bounded for all $n$ large and $H$ is invertible (see, for instance [1, Theorem 2.16]). This allows us to prove that $\Sigma$ does not change substantially under compact perturbation.

Lemma 4. If $M$ is bounded and $K=K^{*}$ is a compact operator, then

$$
\Sigma(M) \cup \operatorname{Spec}_{\operatorname{disc}}(M+K)=\Sigma(M+K) \cup \operatorname{Spec}_{\operatorname{disc}} M .
$$

In particular $\Sigma(M)$ and $\Sigma(M+K)$ coincide outside the real axis.

Proof. The point $z$ is not in the set on the left-hand side if and only if

$$
(M+K-z)^{2}=(M-z)^{2}+\left[(M-z) K+K(M-z)+K^{2}\right]
$$

is invertible and

$$
\left\|\left[P_{n(j)}(M-z)^{2} \mid \mathcal{L}_{n(j)}\right]^{-1}\right\|
$$

is uniformly bounded for some suitable subsequence $n(j)$. According to the above observation, the latter is equivalent to having $(M-z)^{2}$ invertible and

$$
\left\|\left[P_{n(j)}(M+K-z)^{2} \mid \mathcal{L}_{n(j)}\right]^{-1}\right\|
$$

uniformly bounded, so therefore it is equivalent to $z$ not being in the set on the right-hand side. 


\section{Proof of Theorem 1}

We start by noting that condition $(\mathrm{H})$ ensures that

$$
\sigma_{n}(\lambda) \rightarrow 0 \quad \text { as } \quad n \rightarrow \infty .
$$

Indeed, take $v=P_{n} \psi$. Then

$$
\begin{aligned}
\sigma_{n}(\lambda) & \leq \frac{\left\|P_{n}(M-\lambda)^{2} P_{n} \psi\right\|}{\left\|P_{n} \psi\right\|} \\
& =\frac{\left\|P_{n} M^{2} P_{n} \psi-2 \lambda P_{n} M P_{n} \psi+\lambda^{2} P_{n} \psi\right\|}{\left\|P_{n} \psi\right\|} \rightarrow 0
\end{aligned}
$$

as $n \rightarrow \infty$.

Decompose

$$
\begin{gathered}
\mathcal{H}=\mathcal{E} \oplus \mathcal{E}^{\perp}, \\
\mathcal{E}=\operatorname{Span}\{\psi \in \operatorname{Dom} M: M \psi=\lambda \psi\} \subset \operatorname{Dom} M^{2},
\end{gathered}
$$

where $\operatorname{dim} \mathcal{E}<\infty$, and both $\mathcal{E}$ and $\mathcal{E}^{\perp}$ are invariant under $M$. According to the standard notion, $\mathcal{E}^{\perp}$ invariant under $M$ means that $M x \in \mathcal{E}^{\perp}$ for all $x \in$ $\mathcal{E}^{\perp} \cap \operatorname{Dom} M$. The restriction $M \mid \mathcal{E}$ corresponds to multiplication by $\lambda$. Denote by $P_{\mathcal{E}}$ the orthogonal projection onto $\mathcal{E}$. Put

$$
M=M\left(I-P_{\mathcal{E}}\right)+(\lambda-\mu) P_{\mathcal{E}}+\mu P_{\mathcal{E}}=: A+\mu P_{\mathcal{E}},
$$

where $0 \neq \mu \notin \operatorname{Spec} M$ and $\operatorname{Dom} A=\operatorname{Dom} M$. Then $A=A^{*}, M$ is a finite rank perturbation of $A$ and

$$
\operatorname{Spec} A=\{\lambda-\mu\} \cup \operatorname{Spec} M \backslash\{\lambda\} .
$$

For $z \in \mathbb{C}$, let $A(z):=(A-z)^{2}$ with $\operatorname{Dom} A(z)=\operatorname{Dom} M^{2}$. Then $A(z)$ is a holomorphic family of type A for $z \in \mathbb{C}$. If $K(z)=\mu(2 \lambda-2 z-\mu) P_{\mathcal{E}}$, then

$$
(M-z)^{2} x=A(z) x+K(z) x, \quad x \in \operatorname{Dom} M^{2} .
$$

Put $A_{n}(z):=P_{n} A(z) \mid \mathcal{L}_{n}$ and $K_{n}(z)=P_{n} K(z) \mid \mathcal{L}_{n}$.

The validity of Theorem 1 can be formally justified by the following observation. For $z$ close to $\lambda$, $\operatorname{Spec} A(z)$ is the spectrum of $A$ "bent" to the right half-plane by the map $w \mapsto(w-z)^{2}$. Since the numerical ranges of $A_{n}(z)$ are far from zero (cf. Lemma 5), $\left\|A_{n}(z)^{-1}\right\|$ are uniformly bounded for all such $z$. When we add $K(z)$, the truncations are not sectorial any longer, but since $K(z)$ is of finite rank, for $n$ large, $A_{n}(z)+K_{n}(z)$ are still invertible, except for a few isolated points which correspond to perturbations of the isolated eigenvalue $\lambda$ of $A(z)+K(z)$. When some of these isolated points are equal to zero, $z \in \operatorname{Spec}_{2}\left(M \mid \mathcal{L}_{n}\right)$.

Lemma 5. Let $\delta>0$ be small enough. Then there exist nonnegative constants $\alpha, \beta$ and $b$, such that

$$
\operatorname{Num} A_{n}(z) \subseteq \operatorname{Conv}\left[([\alpha, \infty)+i[-\beta, \beta])^{2}\right] \subset\{\operatorname{Re}(z) \geq b>0\}
$$

for all $n \in \mathbb{N}$ and $z \in\{|z-\lambda| \leq \delta\}$.

Proof. By substituting $A$ for $M$ in (4), clearly $\operatorname{Num} A_{n}(z) \subseteq \operatorname{Num} A(z)$ for all $n \in \mathbb{N}$. Since $A=A^{*}$, then $A(z)$ is a normal operator for all $z \in \mathbb{C}$, so that

$$
\operatorname{Num} A(z) \subseteq \overline{\operatorname{Conv}\left[\operatorname{Spec}(A-z)^{2}\right]}, \quad z \in \mathbb{C} .
$$


Since $\lambda \notin \operatorname{Spec} A$, we can find $\delta>0$ small enough, such that $\{|z-\lambda| \leq \delta\}$ does not intersect $\operatorname{Spec} A$. Then there are $\alpha, \beta>0$, such that

$$
\operatorname{Spec}(A-z) \subset((-\infty,-\alpha]+i[-\beta, \beta]) \cup([\alpha, \infty)+i[-\beta, \beta])
$$

for $|z-\lambda| \leq \delta$. Hence

$$
\operatorname{Spec}(A-z)^{2} \subset([\alpha, \infty)+i[-\beta, \beta])^{2} .
$$

The $b>0$ can be found by choosing $\delta>0$ small enough, such that $\beta$ is also small.

Lemma 6. Let $0 \neq \psi \in \operatorname{Dom} H$ be such that $H \psi=\nu \psi$ and $H_{n} P_{n} \psi \rightarrow \nu \psi$. If $H$ and $H_{n}$ are invertible, and $\left\|H_{n}^{-1}\right\| \leq c$ where $c>0$ is independent of $n$, then

$$
\left\|H_{n}^{-1} P_{n} \psi-H^{-1} \psi\right\| \rightarrow 0
$$

as $n \rightarrow \infty$.

Proof.

$$
\left\|H_{n}^{-1} P_{n} \psi-H^{-1} \psi\right\| \leq c\left\|P_{n} \psi-\nu^{-1} H_{n} P_{n} \psi\right\| \rightarrow 0
$$

as $n \rightarrow \infty$.

It is well known that for any bounded operator $H$ and $w \notin \operatorname{Num} H$,

$$
\left\|(H-w)^{-1}\right\| \leq \operatorname{dist}(w, \text { Num } H)^{-1}
$$

(cf. for instance [8, p. 268]). Then according to Lemma [5 for all $n \in \mathbb{N}$ and $|z-\lambda|<\delta, A_{n}(z)$ are invertible and there exists $c_{1}>0$ independent of $n$ and $z$, such that $\left\|A_{n}(z)^{-1}\right\|<c_{1}$. Since $\mathcal{E}$ is an eigenspace of $A(z),(\mathrm{H})$ ensures that $A_{n}(z) P_{n} \psi \rightarrow A(z) \psi$ as $n \rightarrow \infty$ for all $\psi \in \mathcal{E}$. Since $z \notin \operatorname{Spec} A, A(z)$ is invertible. Therefore Lemma 6 yields

$$
\left\|A_{n}(z)^{-1} P_{n} \psi-A(z)^{-1} \psi\right\| \rightarrow 0, \quad \psi \in \mathcal{E},
$$

for all $|z-\lambda|<\delta$.

The various constants $c_{j}>0$ that appear below are independent of $z$ and $n$.

In order to prove Theorem 1, we show that for $\delta>0$ small enough, we can find constants $c>0$ and $\tilde{N}>0$ uniform in $z$, such that $\sigma_{n}(z)^{-1} \leq c$ for all $|z-\lambda|=\delta$ and $n \geq \tilde{N}$. By virtue of (6) and by applying Lemma 2 , the above ensures the existence of $N>0$ such that we can find

$$
z_{n} \in\{|z-\lambda|<\delta\} \cap \operatorname{Spec}_{2}\left(M \mid \mathcal{L}_{n}\right)
$$

for all $n \geq N$.

Let $\delta>0$ be small enough to ensure that Lemma 5 holds and

$$
\operatorname{Spec} M \cap\{|z-\lambda| \leq \delta\}=\{\lambda\} .
$$

Denote by

$$
N_{\delta}:=\{z \in \mathbb{C}:|z-\lambda|=\delta\} .
$$

Since $A(z)+K(z)=(M-z)^{2}$ is invertible, for all $z \in N_{\delta}$,

$$
I+A(z)^{-1} K(z)=A(z)^{-1}(A(z)+K(z))
$$

is also invertible. Since the function $\left\|\left[I+A(\cdot)^{-1} K(\cdot)\right]^{-1}\right\|: N_{\delta} \longrightarrow(0, \infty)$ is continuous, it should be uniformly bounded. Put $c_{2}>0$ such that

$$
\left\|\left[I+A(z)^{-1} K(z)\right]^{-1}\right\| \leq c_{2}^{-1}, \quad z \in N_{\delta} .
$$


Let $\varepsilon>0$ and $z \in N_{\delta}$. Since $N_{\delta}$ is compact, there exists a finite set of points $\left\{w_{j}\right\} \subset N_{\delta}$ satisfying the following property. Given any $z \in N_{\delta}$, there is $w \in\left\{w_{j}\right\}$ such that

$$
\left\|A(z)^{-1}-A(w)^{-1}\right\|<\varepsilon \quad \text { and } \quad|w-z|<\varepsilon .
$$

Since $\operatorname{dim} \mathcal{E}<\infty$ and $\left\{w_{j}\right\}$ is finite, by virtue of (77), there exists $\tilde{N}>0$ such that

$$
\left\|A_{n}(w)^{-1} P_{n} \psi-A(w)^{-1} \psi\right\|<\varepsilon,
$$

for all $n \geq \tilde{N}, w \in\left(w_{j}\right)$ and $\psi \in \mathcal{E}$. Thus, if $w$ satisfies (9),

$$
\begin{aligned}
&\left\|A_{n}(z)^{-1} P_{n} \psi-A(z)^{-1} \psi\right\| \\
& \leq\left\|A_{n}(z)^{-1} P_{n} \psi-A_{n}(w)^{-1} P_{n} \psi\right\|+\left\|A_{n}(w)^{-1} P_{n} \psi-A(w)^{-1} \psi\right\| \\
&+\left\|A(w)^{-1} \psi-A(z)^{-1} \psi\right\| \\
&<\left\|A_{n}(z)^{-1} P_{n} \psi-A_{n}(w)^{-1} P_{n} \psi\right\|+2 \varepsilon \\
&=\left\|A_{n}(z)^{-1} A_{n}(w)^{-1}\left(A_{n}(w)-A_{n}(z)\right) P_{n} \psi\right\|+2 \varepsilon \\
& \leq c_{1}^{2}\left\|(w-z) P_{n}[(w+z)-2 A] P_{n} \psi\right\|+2 \varepsilon \\
& \leq|w-z| c_{1}^{2} c_{3}+2 \varepsilon<c_{4} \varepsilon .
\end{aligned}
$$

The existence of $c_{3}$ is ensured by $(\mathrm{H})$. By choosing $\varepsilon$ small enough, we can find $\tilde{N}>0$ independent of $z$, such that

$$
\left\|A_{n}(z)^{-1} P_{n} K(z)-A(z)^{-1} K(z)\right\|<c_{2} / 2, \quad n \geq \tilde{N} .
$$

Let $x \in \mathcal{H}$. By virtue of (8),

$$
\begin{aligned}
c_{2}\left\|P_{n} x\right\| \leq & \left\|\left(I+A(z)^{-1} K(z)\right) P_{n} x\right\| \\
\leq & \left\|\left(I+A_{n}(z)^{-1} P_{n} K(z)\right) P_{n} x\right\| \\
& +\left\|A_{n}(z)^{-1} P_{n} K(z)-A(z)^{-1} K(z)\right\|\left\|P_{n} x\right\| .
\end{aligned}
$$

Hence, for all $n \geq \tilde{N}$,

$$
\begin{aligned}
\left(c_{2} / 2\right)\left\|P_{n} x\right\| & \leq\left\|\left(I+A_{n}(z)^{-1} P_{n} K(z)\right) P_{n} x\right\| \\
& \leq\left\|A_{n}(z)^{-1}\right\|\left\|\left(A_{n}(z)+P_{n} K(z) P_{n}\right) P_{n} x\right\| \\
& \leq c_{1}\left\|P_{n}(M-z)^{2} \mid \mathcal{L}_{n} P_{n} x\right\| .
\end{aligned}
$$

Therefore $P_{n}(M-z)^{2} \mid \mathcal{L}_{n}$ is invertible and

$$
\sigma_{n}(z)^{-1}=\left\|\left(P_{n}(M-z)^{2} \mid \mathcal{L}_{n}\right)^{-1}\right\| \leq 2 c_{1} / c_{2}
$$

when $z \in N_{\delta}$ and $n \geq \tilde{N}$. This ensures the validity of Theorem 1 , 


\section{An EXAMPle of Limiting SET}

In this section we find the uniform limiting set of second order spectra for a very simple model. We choose this example so that the spectral pollution is maximal when we apply the linear method. We also investigate rank one perturbations of this model. These ideas are close to example I of [7] and those in [2].

Let $E \varsubsetneqq(-\pi, \pi]$ be a finite union of semi-open intervals $\left(a_{k}, b_{k}\right]$. Denote by $E^{c}:=(-\pi, \pi] \backslash E$. Let $M f(x):=m(x) f(x)$ be the linear operator of multiplication by the symbol

$$
m(x)= \begin{cases}1 & \text { if } x \in E \\ -1 & \text { if } x \in E^{c}\end{cases}
$$

acting on $\mathcal{H}=L^{2}(-\pi, \pi)$. Let $\mathcal{L}_{n}:=\operatorname{Span}\left\{e^{-i n x}, \ldots, e^{i n x}\right\}$. Then $M_{n}$ is the $(2 n+1) \times(2 n+1)$ Toeplitz matrix $\left(M_{n}\right)_{j, k}=\hat{m}(j-k)$, where $\hat{m}$ denotes the Fourier coefficients of $m$. Note that $\operatorname{Spec} M=\operatorname{Spec}_{\text {ess }} M=\{ \pm 1\}$.

Denote by $T(m)$ the Toeplitz operator acting on $l^{2}(\mathbb{N})$ whose symbol is $m$, i.e., $T(m)$ has an infinite matrix representation of which the $M_{n}$ are simple truncations. By invoking Gohberg's theorem for piecewise continuous symbols (cf., for instance [1. Theorem 1.23]), we realize that $\operatorname{Spec} T(m)=[-1,1]$. The truncation of $T(m)$ to the subspace

$$
\tilde{\mathcal{L}_{n}}=\operatorname{Span}\left\{\delta_{1}, \ldots, \delta_{2 n+1}\right\} \subset l^{2}(\mathbb{N}),
$$

where $\delta_{k}(n)=\delta_{k, n}$ is the Kronecker delta symbol, equals $M_{n}$ above. By virtue of a result due to Szegö (cf. [1, Theorem 5.14]), for each $\mu \in[-1,1]$ there is a sequence $\mu_{n} \in \operatorname{Spec} M_{n}$ such that $\mu_{n} \rightarrow \lambda$ as $n \rightarrow \infty$. Thus, if we choose the linear method with $\mathcal{L}_{n}$ as approximating subspaces, each point in the spectral gap $(-1,1)$ of $M$ is of spectral pollution.

We now find the limiting set $\Lambda(M)$. According to arguments in the proof of Proposition 3, $\operatorname{Spec}_{2}\left(M \mid \mathcal{L}_{n}\right)$ lies in $\mathbb{T}$ for all $n$. We show that each $\zeta=e^{i \theta},-\pi<$ $\theta \leq \pi$, is in $\Lambda$. Indeed, by virtue of Gohberg's theorem for piecewise continuous symbols, the spectrum of $T(m-\zeta)^{2}$, the Toeplitz operator associated to the symbol $(m-\zeta)^{2}$, equals the segment

$$
\left\{(1+\zeta)^{2} t+(1-\zeta)^{2}(1-t): 0 \leq t \leq 1\right\}
$$

Let $Q_{n}$ denote the orthogonal projection onto $\tilde{\mathcal{L}_{n}}$. Then

$$
P_{n}(M-z)^{2}\left|\mathcal{L}_{n}=Q_{n} T(m-\zeta)^{2}\right| \tilde{\mathcal{L}}_{n}
$$

Since the essential spectrum of $T(m-\zeta)^{2}$ is a set with no interior, then each point in it can be approximated using a Weyl singular sequence (cf. [6, Theorem 10.10]). In particular, by taking $t=(1-\cos \theta) / 2$, so is the origin. Hence for all $k>0$, there is $\psi_{k} \in l^{2}(\mathbb{N}),\left\|\psi_{k}\right\|=1$, such that $\left\|T(m-\zeta)^{2} \psi_{k}\right\|<1 / k$. Since

$$
\sigma_{n}(\zeta) \leq \frac{\left\|Q_{n} T(m-\zeta)^{2} Q_{n} \psi_{k}\right\|}{\left\|Q_{n} \psi_{k}\right\|} \rightarrow\left\|T(m-\zeta)^{2} \psi_{k}\right\|
$$

as $n \rightarrow \infty$, then $\zeta \in \Sigma$. Consequently Proposition 3 ensures that $\Lambda(M)=\mathbb{T}$.

Let us now discuss how the points of $\operatorname{Spec}_{2}\left(M \mid \mathcal{L}_{n}\right)$ distribute along $\mathbb{T}$. We saw in Section 2 that

$$
z \in \operatorname{Spec}_{2}\left(M \mid \mathcal{L}_{n}\right) \quad \text { iff } \quad \frac{z+z^{-1}}{2} \in \operatorname{Spec} M_{n}
$$


By construction, $M_{n}$ coincides with the truncation of $T(m)$ to the subspace $\tilde{\mathcal{L}}_{n}$. Let

$$
\left\{z_{k}^{(n)}\right\}_{k=1}^{n}:=\operatorname{Spec}_{2}\left(M \mid \mathcal{L}_{n}\right) \cap\{\operatorname{Im} z>0\}
$$

and

$$
w_{k}^{(n)}:=\frac{z_{k}^{(n)}+\bar{z}_{k}^{(n)}}{2} \in(-1,1) .
$$

Then the mean of the points in $\operatorname{Spec}_{2}\left(M \mid \mathcal{L}_{n}\right)$ is

$$
\begin{aligned}
\frac{1}{2 n} \sum_{k=1}^{n} z_{k}^{(n)}+\bar{z}_{k}^{(n)} & =\frac{1}{n} \sum_{k=1}^{n} w_{k}^{(n)}=\frac{1}{n} \operatorname{Tr} M_{n} \\
& =\hat{m}(0)=\frac{1}{2 \pi}\left(|E|-\left|E^{c}\right|\right)
\end{aligned}
$$

for all $n$. Here $|\cdot|$ denotes the Lebesgue measure.

A version of Szegö's first limit theorem for Toeplitz operators [1, Theorem 5.10] permits us to say even more about the localization of $\operatorname{Spec}_{2}\left(M \mid \mathcal{L}_{n}\right)$ in the limit $n \rightarrow \infty$. According to [1, Corollary 5.12], for each Borel set $B \subset[-1,1]$,

$$
\frac{1}{n} \sum_{w_{k}^{(n)} \in B} 1 \rightarrow \frac{|\{x: m(x) \in B\}|}{2 \pi} .
$$

Then for all $0<\varepsilon<1$,

$$
\frac{1}{n} \sum_{w_{k}^{(n)} \in[-1,-1+\varepsilon]} 1 \rightarrow \frac{\left|E^{c}\right|}{2 \pi} \text { and } \frac{1}{n} \sum_{w_{k}^{(n)} \in[1-\varepsilon, 1]} 1 \rightarrow \frac{|E|}{2 \pi}
$$

as $n \rightarrow \infty$. Consequently, although each point in $\mathbb{T}$ is in the limiting set $\Lambda$, for $n$ large almost $100 \cdot|E| /(2 \pi)$ percent of the points in $\operatorname{Spec}_{2}\left(M \mid \mathcal{L}_{n}\right)$ cluster near 1 and the other $100 \cdot\left|E^{c}\right| /(2 \pi)$ percent cluster near -1 .

In order to envisage the numerical scope of Theorem 1 we can test it against numerical data. For this we consider rank one perturbations of the above model. Let $g \in \mathcal{H}$ be a fixed vector such that $\|g\|=1$, and let $K f:=\langle f, g\rangle g$ for all $f \in \mathcal{H}$. We first explicitly compute the discrete spectrum of $M+K$.

TABle 1. Estimation of the isolated eigenvalues of $M+K$ for $g(x)=1$ and $E=(0, \pi]$. The first column corresponds to the theoretical value.

\begin{tabular}{|c|c|c|c|c|}
\hline$\lambda$ & $n$ & $\operatorname{Re} z_{n}-\left|\operatorname{Im} z_{n}\right|$ & $\operatorname{Re} z_{n}+\left|\operatorname{Im} z_{n}\right|$ & $\operatorname{Re} z_{n}-\lambda$ \\
\hline-0.61803398 & 85 & -0.64711164 & -0.59156988 & 0.00130677 \\
& 120 & -0.64232258 & -0.59559824 & 0.00092642 \\
& 155 & -0.63930167 & -0.59820046 & 0.00071708 \\
& 190 & -0.63717720 & -0.60006016 & 0.00058469 \\
& 225 & -0.63557976 & -0.60147516 & 0.00049347 \\
\hline 1.61803398 & 85 & 1.58929960 & 1.64481953 & 0.00097441 \\
& 120 & 1.59398716 & 1.64069975 & 0.00069052 \\
& 155 & 1.59695260 & 1.63804631 & 0.00053453 \\
& 190 & 1.59904216 & 1.63615390 & 0.00043595 \\
& 225 & 1.60061565 & 1.63471625 & 0.00036803 \\
\hline
\end{tabular}


TABLE 2. Estimation of the isolated eigenvalues of $M+K$ for $g(x)=1$ and $E=(-15 \pi / 16, \pi]$. The first column corresponds to the theoretical value.

\begin{tabular}{|c|c|c|c|c|}
\hline$\lambda$ & $n$ & $\operatorname{Re} z_{n}-\left|\operatorname{Im} z_{n}\right|$ & $\operatorname{Re} z_{n}+\left|\operatorname{Im} z_{n}\right|$ & $\operatorname{Re} z_{n}-\lambda$ \\
\hline-0.97901994 & 85 & -0.99169545 & -0.97384630 & 0.00375093 \\
& 120 & -0.98897219 & -0.97406728 & 0.00249979 \\
& 155 & -0.98740174 & -0.97435952 & 0.00186068 \\
& 190 & -0.98635662 & -0.97465104 & 0.00148388 \\
& 225 & -0.98561863 & -0.97491625 & 0.00124750 \\
\hline 1.97901994 & 85 & 1.95326913 & 2.00377483 & 0.00049795 \\
& 120 & 1.95700470 & 2.00030477 & 0.00036520 \\
& 155 & 1.95961155 & 1.99784090 & 0.00029371 \\
& 190 & 1.96164380 & 1.99591836 & 0.00023886 \\
& 225 & 1.96314714 & 1.99449943 & 0.00019665 \\
\hline
\end{tabular}

Lemma 7. Let $H$ be self-adjoint and such that $\operatorname{Spec} H=\operatorname{Spec}_{\mathrm{ess}} H$. Then the isolated eigenvalue of finite multiplicity of $H+K$ are nondegenerate and they are the nonzero solutions $\lambda$ of

$$
\left\langle(\lambda-H)^{-1} g, g\right\rangle=1 .
$$

Proof. If $(H+K-\lambda) \psi=0$ for $\psi \neq 0$ and $\lambda \notin \operatorname{Spec} H$, then

$$
(H-\lambda) \psi+\langle\psi, g\rangle g=0 .
$$

If we normalize by $\langle\psi, g\rangle=1$, then $\psi=(\lambda-H)^{-1} g$. A substitution in the normalizing identity yields (11). Conversely suppose that (11) holds. By putting $\psi:=(\lambda-H)^{-1} g$, we achieve $(H+K-\lambda) \psi=0$.

Hence, $\lambda \neq \pm 1$ is an eigenvalue of $M+K$ iff

$$
\frac{\mu_{g}(E)}{\lambda-1}+\frac{\mu_{g}\left(E^{c}\right)}{\lambda+1}=1,
$$

where $\mu_{g}(B)=(2 \pi)^{-1} \int_{B}|g|^{2} \mathrm{~d} x$.

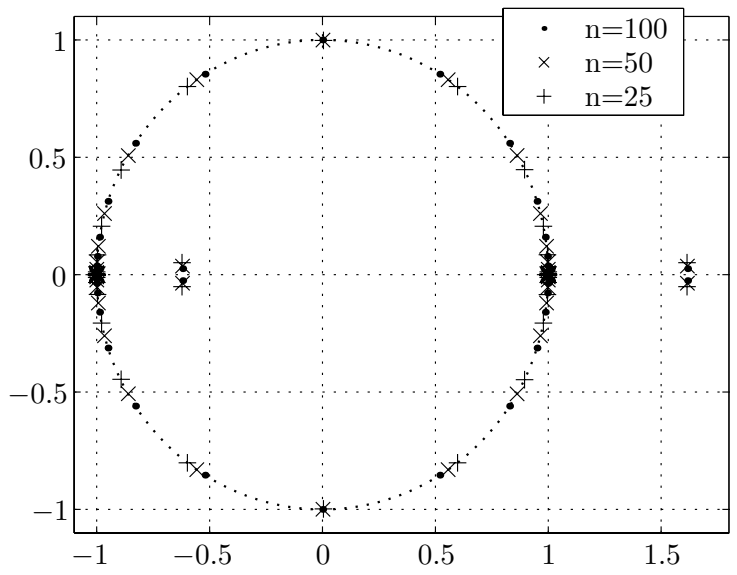

FiguRE 1. $\operatorname{Spec}_{2}\left(M+K \mid \mathcal{L}_{n}\right)$ for three values of $n$. The operator corresponds to $g(x)=1$ and $E=(0, \pi]$. See Table 1 . 


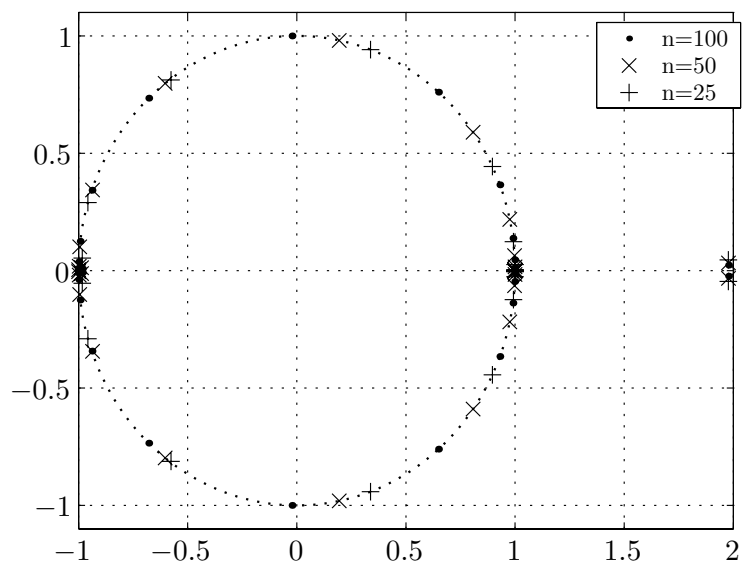

FiguRE 2. $\operatorname{Spec}_{2}\left(M+K \mid \mathcal{L}_{n}\right)$ for three values of $n$. The operator corresponds to $g(x)=1$ and $E=(-15 \pi / 16, \pi]$. See Table 2 .
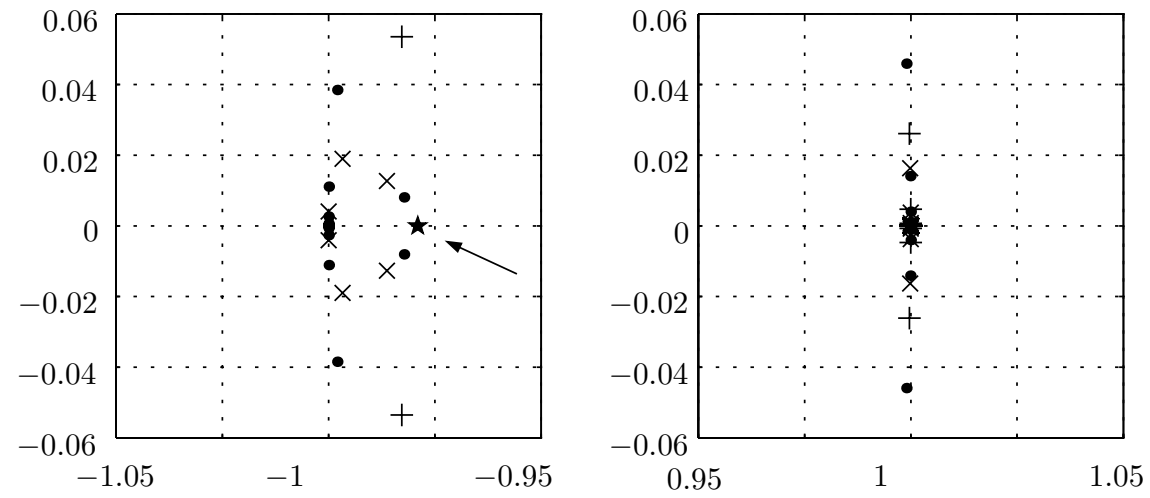

Figure 3. Two zoom pictures of Figure 2, clustering near \pm 1 .

The star shows the location of the theoretical eigenvalue.

In Tables 1 and 2 we show some numerical outputs produced by using the method of second order spectra applied to $M+K$. We found $z_{n}$ by adopting the algorithms for computing second order spectra available at the internet address mentioned in [7. This value corresponds to the point in $\operatorname{Spec}_{2}\left(M+K \mid \mathcal{L}_{n}\right)$, closest to the isolated nondegenerate eigenvalue $\lambda$. We found the theoretical value of $\lambda$ by using (12). In both tables it is remarkable that although bound (2) only provides estimation for the first digit of $\lambda$ at $n=225$, the actual value of $\operatorname{Re} z_{n}$ is correct up to three digits. For Table 2 we chose an extreme case where one of the non-degenerate eigenvalues is close to the point -1 of the essential spectrum. It is remarkable that the even the step $n=85$, which only takes a few seconds to run on a PC, already detects the presence of this eigenvalue.

Figures 1, 2] and 3] show how the rest of the second order spectrum in these two cases distribute along the complex plane for three different values of $n$. Note that the clustering predicted for the unperturbed case seems to be largely kept with the exception of some few points that approximate the isolated eigenvalues. 


\section{The COndition $(\mathrm{H})$}

As in the linear case, an effective numerical implementation of the method of second order spectra to unbounded operators demands verifying $(\mathrm{H})$ without having much information about the eigenfunctions $\psi$. This is achieved sometimes by means of a perturbative reasoning. We do not claim that this is easy in general, but in some situations the following standard argument can be useful.

The general strategy involves a dominating operator $X$, which has compact resolvent and a complete set of eigenfunctions that can be found explicitly. Both operators $M^{k}, k=1,2$, should be relatively $X$-bounded, in the sense that $\operatorname{Dom} X \subseteq$ Dom $M^{2}$ and

$$
\left\|M^{k} u\right\| \leq a_{k}\|X u\|+b_{k}\|u\|, \quad u \in \operatorname{Dom} X,
$$

for uniform constants $a_{k}>0$ and $b_{k}>0$. The best $a_{k}$, such that the above holds for some $b_{k}$, is called a relative bound. Let $\left\{\phi_{n}\right\}_{n=1}^{\infty}$ be the set of eigenfunctions of $X$. If the eigenfunction $\psi$ of $M$ associated to the eigenvalue $\lambda$ lies in $\operatorname{Dom} X$, by virtue of the spectral theorem, $X$ and $P_{n}$ commute, and hence

$$
\left\|X P_{n} \psi-X \psi\right\|=\left\|P_{n} X \psi-X \psi\right\| \rightarrow 0
$$

as $n \rightarrow \infty$. Then $\left\|M^{k} P_{n} \psi-M^{k} \psi\right\| \rightarrow 0$, ensuring (H).

The following result illustrates this strategy. Recall that the class $K_{1}$ consists of all $V: \mathbb{R} \longrightarrow \mathbb{R}$ such that

$$
\sup _{x \in \mathbb{R}} \int_{|x-y| \leq 1}|V(y)| \mathrm{d} y<\infty .
$$

Theorem 8. Let $\phi_{n}$ be the eigenfunctions of the harmonic oscillator $\left(-\partial_{x}^{2}+x^{2}\right)$ acting on $L^{2}(\mathbb{R})$. Let $V: \mathbb{R} \longrightarrow \mathbb{R}$ be such that

$$
V \in\left[K_{1} \cap W^{2,2}(\mathbb{R})\right]+W^{\infty, 2}(\mathbb{R}) .
$$

Then $(\mathrm{H})$ holds for every eigenvalue and eigenfunction of the operator $M=-\partial_{x}^{2}+V$ acting on $L^{2}(\mathbb{R})$.

Analogous results can be found for higher dimensions.

Proof. Since $V \in L^{2}+L^{\infty}$, multiplication by $V$ is relatively $\partial_{x}^{2}$-bounded with bound 0 (cf. [3, §1.2]), hence $M$ is relatively $\partial_{x}^{4}$-bounded with bound 0 .

We prove that $M^{2}$ is relatively $\partial_{x}^{4}$-bounded with bound 1 . For this, note that

$$
\left(-\partial_{x}^{2}+V\right)^{2} u=\partial_{x}^{4} u-2 V \partial_{x}^{2} u-2 V^{\prime} \partial_{x} u+\left(V^{2}-V^{\prime \prime}\right) u .
$$

Since multiplication by $V$ is $\partial_{x}^{2}$-bounded with relative bound 0 , then $2 V \partial_{x}^{2}$ is $\partial_{x}^{4}$ bounded with relative bound 0 . Since $V^{\prime} \in L^{2}+L^{\infty}$, a similar reasoning ensures that $2 V^{\prime} \partial_{x}$ is $\partial_{x}^{3}$-bounded with relative bound 0 . Since $V^{\prime \prime}$ lies in $L^{2}+L^{\infty}$, multiplication by $V^{\prime \prime}$ is $\partial_{x}^{2}$-bounded with relative bound 0 . Since $V^{2}$ is $\partial_{x}^{2} V$-bounded with relative bound 0 and

$$
\partial_{x}^{2} V u=V^{\prime \prime} u+2 V^{\prime} \partial_{x} u+V \partial_{x}^{2} u,
$$

then $V^{2}$ is $\partial_{x}^{4}$-bounded with relative bound 0 . This ensures that $M^{2}$ is $\partial_{x}^{4}$-bounded with relative bound 1 .

By virtue of the Kato-Rellich theorem, we do not have to worry about the domains. Here we are considering

$$
\operatorname{Dom} M=W^{2,2} \quad \text { and } \quad \operatorname{Dom} M^{2}=W^{2,4} .
$$


The mentioned result ensures that $M$ (and hence $M^{2}$ ) are self-adjoint in these domains.

Put $X=\left(-\partial_{x}^{2}+x^{2}\right)^{2}$. Since $V \in K_{1}$, then $|\psi(x)| \leq c e^{-a|x|}$ for some constants $a>0$ and $c>0$ (cf. [15, §C.3]). Together with the inclusion $\psi \in W^{2,4}$, this bound ensures $\psi \in \operatorname{Dom}\left(-\partial_{x}^{2}+x^{2}\right)=W^{2,2} \cap \operatorname{Dom}\left(x^{2}\right)$ and $\left(-\psi^{\prime \prime}+x^{2} \psi\right) \in W^{2,2} \cap \operatorname{Dom}\left(x^{2}\right)$. The only nontrivial facts of the latter assertion are, perhaps, the inclusions $x^{2} \psi \in$ $W^{2,2}$ and $\psi^{\prime \prime} \in \operatorname{Dom}\left(x^{2}\right)$. The first follows from the second by differentiating twice the term $x^{2} \psi$ and noting that $\left(x \psi^{\prime}\right)^{\prime} \in L^{2}$. The second inclusion is achieved by means of the following trick. Since $M \psi$ is eigenvector of $M,\left|-\psi^{\prime \prime}(x)+V(x) \psi(x)\right| \leq$ $c e^{-a|x|}$. Then

$$
\begin{aligned}
\left\|x^{2} \psi^{\prime \prime}\right\| & \leq\left\|x^{2}\left(-\psi^{\prime \prime}+V \psi\right)\right\|+\left\|x^{2} V \psi\right\| \\
& \leq c_{1}+\left(\int x^{4}|V(x)|^{2}|\psi(x)|^{2} \mathrm{~d} x\right)^{1 / 2} \\
& \leq c_{1}+c_{2}\left(\int x^{4} e^{-a|x|}|V(x)|^{2} \mathrm{~d} x\right)^{1 / 2} .
\end{aligned}
$$

The latter integral is bounded because of $V \in L^{2}+L^{\infty}$, therefore $\psi^{\prime \prime} \in \operatorname{Dom}\left(x^{2}\right)$. Thus $\psi \in \operatorname{Dom} X$.

Finally we show that $\partial_{x}^{4}$ is $\left(\partial_{x}^{2}+x^{2}\right)^{2}$-bounded. For this, let

$$
A:=\frac{1}{2}\left(x+\partial_{x}\right) \quad \text { and } \quad A^{*}=\frac{1}{2}\left(x-\partial_{x}\right) .
$$

Then $\left(-\partial_{x}^{2}+x^{2}\right)=4 A A^{*}-1$ and $\partial_{x}^{4}=\left(A-A^{*}\right)^{4}$. Thus the desired property follows from the identity (cf. [12, eq. (X.28)])

$$
\left\|A^{\#_{1}} \cdots A^{\#_{n}} u\right\| \leq c\left\|\left(-\partial_{x}^{2}+x^{2}\right)^{n / 2} u\right\|, \quad n=1,2, \ldots,
$$

where $A^{\#_{k}}$ is either $A$ or $A^{*}$. This is easily shown by induction and using the estimate

$$
\left\|\left(-\partial_{x}^{2}+x^{2}\right)^{k / 2} u\right\| \leq\left\|\left(-\partial_{x}^{2}+x^{2}\right)^{n / 2} u\right\|, \quad k<n .
$$

This completes the proof of the theorem.

In the above result, we have in mind the case $V=W+S$, where $W$ is periodic and $S$ is in $L^{2}(\mathbb{R})+L^{\infty}(\mathbb{R})_{\varepsilon}$. Under this hypothesis, it is well known that the essential spectrum of $M$ has a band gap structure determined solely by $W$, whereas the perturbation $S$ can produce nonempty discrete spectrum (cf., e.g., 13, Chapter XIII.16] and also [10]).

\section{ACKNOWLEDGMENTS}

The author wishes to thank E.B. Davies, E. Shargorodsky and V. Strauss for their useful comments during the preparation of this manuscript. The author was partially supported by the Pacific Institute for the Mathematical Sciences.

\section{REFERENCES}

[1] A. Bötcher and B. Silbermann, Introduction to large truncated Toeplitz matrices, Springer, New York, 1999. MR1724795 (2001b:47043)

[2] L. Boulton, "Projection methods for discrete Schrödinger operators," Proc. London Math. Soc. 88 (2004) 526-544. MR 2032518 (2005e:47077)

[3] H.L. Cycon, R.G. Froese, W. Kirsch, and B. Simon, Schrödinger operators, Springer, Berlin, 1987. MR0883643 (88g:35003) 
[4] E.B. DAvies, "Spectral enclosures and complex resonances for general self-adjoint operators", LMS J. Comput. Math. 1 (1998) 42-74. MR1635727 (2000e:47043)

[5] E.B. Davies and M. Plum, "Spectral pollution", IMA J. Numer. Anal. 24 (2004) 417-438. MR2068830 (2005c:47027b)

[6] P. Hislop AND I. Sigal, Introduction to Spectral Theory, Springer, New York, 1996. MR1361167 (98h:47003)

[7] M. LEVITIN AND E. Shargorodsky, "Spectral pollution and second order relative spectra for self-adjoint operators", IMA J Numer. Anal. 24 (2004) 393-416. MR2068829|(2005c:47027a)

[8] T. Kato, Perturbation theory for linear operators, 2nd edition, Springer-Verlag, Berlin, 1980. MR0407617 (53:11389)

[9] T. Kato, "On the upper and lower bounds on eigenvalues", J. Phys. Soc. Japan 4 (1949) 334-339. MR0038738 (12:447b)

[10] A. KisELev, "Stability of the absolutely continuous spectrum of Schrödinger operators under slowly decaying perturbations and a.e. convergence of integral operators", Duke Mathematical Journal, 94 (1998) 619-649. MR1639550 (99h:34124)

[11] M. Reed And B. Simon, Methods of modern mathematical physics, volume 1: Functional Analysis, Academic Press, New York, 1980. MR0751959 (85e:46002)

[12] M. Reed And B. Simon, Methods of modern mathematical physics, volume 2: Selfadjointness, Academic Press, New York, 1975. MR0493420 (58:12429b)

[13] M. Reed And B. Simon, Methods of modern mathematical physics, volume 4: analysis of operators, Academic press, New York, 1978. MR0493421 (58:12429c)

[14] E. Shargorodsky, "Geometry of higher order relative spectra and projection methods", $J$. Oper. Theo. 44 (2000) 43-62. MR1774693 (2001f:47004)

[15] B. Simon, "Schrödinger semigroups", Bull. Am. Math. Soc. 7 (1982) 447-526. MR0670130 (86b:81001a)

Department of Mathematics, Heriot-Watt University, Riccarton, Edinburgh EH14 4AS, UNITED KINGDOM

E-mail address: lyonell@ma.hw.ac.uk 